\title{
Analysis of Error-Agnostic Time- and Frequency-Domain Features Extracted From Measurements of 3-D Accelerometer Sensors
}

\author{
Waltenegus Dargie, Member, IEEE, and Mieso K. Denko, Senior Member, IEEE
}

\begin{abstract}
This paper investigates the expressive power of several time- and frequency-domain features extracted from 3-D accelerometer sensors. The raw data represent movements of humans and cars. The aim is to obtain a quantitative as well as a qualitative expression of the uncertainty associated with random placement of sensors in wireless sensor networks. Random placement causes calibration, location and orientation errors to occur. Different type of movements are considered-slow and fast movements; horizontal, vertical, and lateral movements; smooth and jerky movements, etc. Particular attention is given to the analysis of the existence of correlation between sets of raw data which should represent similar or correlated movements. The investigation demonstrates that while frequency-domain features are generally robust, there are also computationally less intensive time-domain features which have low to moderate uncertainty. Moreover, features extracted from slow movements are generally error prone, regardless of their specific domain.
\end{abstract}

Index Terms-Accelerometer sensors, feature extraction, frequency-domain features, measurement errors, time-domain features, wireless sensor networks.

\section{INTRODUCTION}

$\mathbf{T}$ HIS paper examines an essential assumption based on which a large number of self-organizing and communication protocols in wireless sensor networks are developed: namely, nodes are deployed randomly. The assumption is plausible for some reasons. For example, in a rescue operation, one may not be able to carefully place sensor nodes, given the urgency of the operation. Likewise, in a health care application that monitors the activities of nurses, the nurses may not be able to pay much attention as to how the nodes are placed in some parts of their body (lower arm, upper arm; lower leg, thigh; back, shoulder, etc.). The assumption, however, should not entirely ignore placement and orientation errors and their impact on the quality of sensed data. One way to go around this problem is to have dense deployment, so that by taking measurements from a large number of closely placed nodes,

Manuscript received September 02, 2009; revised November 15, 2009; accepted November 15, 2009. Date of publication February 02, 2010; date of current version April 07, 2010.

W. Dargie is with the Chair of Computer Networks, Technical University of Dresden, 01062, Dresden, Germany (e-mail: waltenegus.dargie@tu-dresden. de).

M. K. Denko is with the School of Computer Science, University of Guelph, Guelph, ON N1G 2W1 Canada (e-mail: denko@cis.uoguelph.ca).

Color versions of one or more of the figures in this paper are available online at http://ieeexplore.iee.org.

Digital Object Identifier 10.1109/JSYST.2009.2039735 these errors can be minimized. Dense deployment, however, has its own problem due to cost, management, and computational complexities.

Another approach is to identify error agnostic features that can be extracted from the sensed data. Ideally, these features enable to recognize interesting events in the network with appreciable accuracy regardless of how and where the sensors are placed. Hence, we investigate the robustness of time- and frequency-domain features to calibration, placement, and orientation errors. We focus on cheap and randomly deployed accelerometer sensors that monitor the 3-D movements of humans and cars.

A close scrutiny into existing or proposed applications for wireless sensor networks reveals that movement (vibration) sensors are widely employed. For instance, accelerometer sensors are used to monitor the integrity of structures (bridges and building) [1]; transportation infrastructures [2]; supply-chain management [3]; Healthcare [4]; and active volcano [5]. Almost all of these applications employ model-based digital signal processing to detect interesting events such as defects in structures, abnormal drives, and damage in wheels.

We shall demonstrate that even though frequency-domain features are in general error agnostic, there are also simple and straightforward time-domain features that can be useful to many practical applications. The computational complexity of time domain features is significantly lower and can be carried out locally, on the wireless sensor nodes. Another interesting observation is that slow movements are error prone and difficult to recognize both with time- and frequencydomain features.

The contribution of this paper is summarized as follows.

1) Whereas there is a significant body of work on accelerometer sensors, to the best of our knowledge, this is the first comprehensive work that investigates the expressiveness of a large set of time- and frequency-domain features.

2) A qualitative metric based on fuzzy-sets and fuzzy membership functions that are defined and employed to examine the robustness of the features considered.

The remaining part of this paper is organized as follows. In Section II, related work is summarized. In Section III, the timeand frequency-domain features that are used in our analysis are discussed. In Section IV, the methodology to acquire the sensor data is presented; and the description of the scenarios for data collection is given. In Section V, a detail account of the analysis as well as its results are discussed. Finally, in Section VI, concluding remarks and outlook to future work are given. 


\section{RELATED WORK}

A significant body of work exists on energy-efficient protocols for communication and self-organization in wireless sensor networks. Comparatively, the research community has so far focused on three aspects of signal processing, namely, aggregation, sampling, and compression. Dargie et al. [6] and Chao et al. [7] employ a local maxima technique to reduce the network's traffic. Ganesan et al. [8] propose data aggregation and compression mechanisms based on spatial interpolation of data and temporal signal segmentation. Lin proposes a sampling frequency control algorithm and a data compression algorithm [9]—each of them are dependent on the quality of the higher level features extracted from the raw sensor data. The sampling algorithm adjusts the sampling rate based on the features quality. When the sampling frequency cannot be controlled, a data compression algorithm is adopted to reduce the amount of transmitted data. Tang and Raghavendra [10] propose the ESPIHT compression algorithm that uses a distributed source coding and exploits spatio-temporal correlation. Bandyopadhyay et al. [11] give analytical results concerning the tradeoffs between sensor density, energy usage, throughput, delay, temporal sampling rates and spatial sampling rates. A more detailed survey concerning the existing data aggregation and information fusion approaches is given by Nakamura et al. [12] and [13]. Likewise, Tang et al. [10] surveys compression techniques.

As far as modeling and processing measurements of accelerometer sensors are concerned, several techniques and features have been considered. Huynh and Schiele [14] recommend a careful selection of features for different activities. Their experiment result suggests that the choice of a feature and a corresponding window length over which the feature is computed affect a recognition rate. Lukowicz et al. [15] investigate the existence of correlation in accelerometer signals to estimate various human activities.

Perhaps the most frequently employed technique in examining accelerometer data is coherence. An interesting work related to this is the one carried out by Engin et al. [16] and [17], in which the presence of correlation between different axes of individual accelerometers and between different segments of the same limb (of a human body) is used to study the characteristics of tremor in patients with Parkinson's disease (PD).

Marin-Perianu et al. [18] experiment with an incremental correlation algorithm that enables wireless sensor nodes to determine whether they are traveling together (in supply chain management). The algorithm is implemented locally on a sensor node and the data processed is a real-time data series. The scalability of the algorithm is tested with respect to complexities related to communication, energy, memory and speed of execution.

The approaches above identify a set of time- and frequencydomain features and adopt a particular technique to recognize various activities; and to examine the existence of correlation between these activities. Except for Huynh and Schiele, who show how a recognition rate can be affected by the choice of features and their window length, the rest focus rather on the modeling aspect and employ a single technique (usually the coherence function) to recognize activities. These approaches, how-
TABLE I

Time- AND FREQUenCy-Domain FeATURES TO ANALYZE DATA FROM ACCELEROMETER SENSORS

\begin{tabular}{|l|l|}
\hline Domain & Feature \\
\hline \hline Time & $\begin{array}{l}\text { Mean, zero crossing rate, mean-value crossing rate, } \\
\text { maxima/minima, autocorrelation, cross correlation, } \\
\text { linear correlation coefficient, standard deviation }\end{array}$ \\
\hline Frequency & $\begin{array}{l}\text { Energy, correlation (for FFT and STFT), } \\
\text { spectral roll-off, spectral centroid, spectral flux }\end{array}$ \\
\hline
\end{tabular}

ever, do not reveal sufficient insight about the robustness of the features employed. We build upon the existing approaches, but place our focus on investigating the robustness of the features to measurement errors.

\section{MAIN FeATURES}

The time- and frequency-domain features we consider are listed in Table I. These features are used by many of the applications listed in Section I to recognize the occurrence of interesting phenomena. The time domain features capture and express temporal aspects, while the frequency-domain features capture and express spectral aspects. The extraction of time domain features does not require intensive pre-processing, but requires that transmission errors (noise and packet-loss) should be accounted for. Moreover, the comparison of two or more time-series measurements requires that the measurements are synchronized in time. On the other hand, the frequency-domain features are robust to transmission errors, but require intensive pre-processing [framing, windowing, filtering, and fast Fourier transformation (FFT)]. Hence, there is a tradeoff between the cost of feature extraction and the robustness of the features.

In the next subsections, a brief summary of the features listed in Table $\mathrm{I}$ is given.

\section{A. Time-Domain Features}

1) Zero-Crossing: This reveals how often a signal (measurement) crosses a zero-reference line. It is a direct indication of the fundamental frequency of the signal. If the calibration position is known, the zero-crossing rate can be used to estimate the orientation of an accelerometer sensor. For example, if a sensor is calibrated by standing it up (say, along the $y$-axis), then it will produce an acceleration of $1 \mathrm{~g}$ if it is laid flat with a displacement of $90^{\circ}$ either in the $z$-axis or in the $x$-axis. The zero-crossing rate is expressed as

$$
Z C R(s)=\frac{1}{N} \sum_{i=0}^{N-1} F(s[i] \cdot s[i-1]<0)
$$

where $s$ is a discrete, time-series sequence and $s[i]$ and $s[i-1]$ are the $i$ th and $(i-1)$ th sample values. $F=1$ if the evaluation is true, $F=0$ otherwise. Sensors which have different calibration will apparently have different zero-reference line. Therefore, it is difficult to compare their time-series measurements. To avoid this problem, the mean-value crossing rate is used, in which case a calibration-sensitive threshold is defined as $\bar{S}=1 / N \sum_{i=0}^{N} s_{1}[i]$. 
2) Correlation Coefficient: This is a measure of the existence of a linear dependency between two time-series measurements. It takes the quotients of the covariance and variance of the individual measurements into account

$$
C C\left(s_{1}, s_{2}\right)=\frac{\operatorname{COV}\left(s_{1}, s_{2}\right)}{\sqrt{\operatorname{VAR}\left(s_{1}\right) \operatorname{VAR}\left(s_{2}\right)}}
$$

where $\operatorname{COV}\left(s_{1}, s_{2}\right)=(1 / N) \sum_{i=1}^{N}\left(s_{1}[i]-\bar{S}_{1}\right)^{2}\left(s_{2}[i]-\bar{S}_{2}\right)^{2}$ and, $\operatorname{VAR}(s)=(1 / N) \sum_{i=1}^{N}(s[i]-\bar{S})^{2}$.

3) Cross Correlation: Is an indication of the existence of a correlation between two time series measurements $s_{1}[i]$ and $s_{2}[i]$, where $s_{1}$ and $s_{2}$ may represent either the same type of movement measured at different locations, or a single movement measured at the same location but at different times. In case $s_{2}(t)$ represents $s_{1}(t+\tau)$, where $\tau$ is a specified time lag, the two variables are usually not statistically independent, and large cross correlations between $s_{1}$ and $s_{2}$ can result. Mathematically, the cross correlation, $X C$, is described as follows:

$$
X C(n)=\sum_{\tau=\infty}^{\infty} s_{1}[\tau] s_{2}[n+\tau] .
$$

Similarly, the autocorrelation is the cross correlation of a time-series measurement with itself.

\section{B. Frequency-Domain Features}

In order to extract frequency-domain features, the FFT is computed. It has a computational complexity of $n \log (n)$, where $n$ is the number of samples. It is the fastest transformation process between time and frequency domain. However, the FFT does not reveal how fast the signal's frequency changes over time. Therefore, it is necessary to divide the time-series measurements into different, short-duration windows. In order to avoid frequency leakages at the two edges of each window, it is customary to overlap neighbor frames, as a rule the overlap is $25 \%$ to $50 \%$ [6], [19]. Afterwards, FFT is performed on each of these windows. This process is called Short Time Fourier Transformation (STFT). Once the FFT or STFT coefficients are obtained, various more expressive features can be extracted. Below is a short summary of some of them.

1) Spectral Centroid: This represents the balancing point of the spectral power distribution

$$
C_{t}=\frac{\sum_{n=1}^{N} M_{t}[n] \cdot n}{\sum_{n=1}^{N} M_{t}[n]}
$$

where $M_{t}[n]$ is the magnitude value of the spectrum at position (frequency) $n$.

2) Band Energy: Expresses the energy of the subbands normalized by the total energy of the signal.

3) Spectral Roll-Off (SRO): Measures the frequency below which a certain amount of spectral energy resides. It measures the "skewness" of the spectral shape [20]. Mathematically, it is expressed as

$$
\mathrm{SRO}=\sum_{n=1}^{R_{t}} M_{t}[n] .
$$

The sum of the spectrum up to the roll-off frequency signifies $\alpha \%^{1}$ of the total spectrum.

4) Spectral Flux: This is defined as the difference between the magnitude spectra of successive frames [20]

$$
S F=\sum_{n=1}^{N}\left(N_{t}[n]-N_{t-1}[n]\right)^{2}
$$

where $N_{t}[n]$ is the normalized magnitude value of the $n^{\text {th }}$ position of the $t^{\text {th }}$ frame.

5) Maxima: This measures the similarity in transition of the first $n$ Maxima of an expressive feature. The measurements should have the same number of samples and these samples will be transformed into the frequency domain. The $N$ discrete samples produce $N$ discrete frequency coefficients. Since the Maxima is dependent on the dominant frequency components, they can be considered to contain typical structural characteristics. Equation (7) expresses how the corresponding frequencies can be calculated from the discrete spectrum

$$
\omega_{n}=2 \pi \cdot \frac{n}{(2 N+1)} \cdot \frac{1}{T} .
$$

Hence, two sets of measurements exhibit similarity if their first $n$ Maxima exhibit strong similarities. This applies to the magnitude as well as the position of the Maxima.

\section{MeThodology}

We used SunSpot sensor nodes, each containing three accelerometers that are aligned orthogonally, along the $x-, y$, and $z$-axes. For the detail description of the nodes employed in the experiment, the reader is referred to [21]. The nodes maximum sampling frequency was $350 \mathrm{~Hz}$ (i.e., maximum sampling at $\approx 3 \mathrm{~ms}$ ). The average communication delay between the sensor nodes and the remote sink was $4 \mathrm{~ms}$. To minimize packet loss, all data are logged to a remote computer at an average frequency of $150 \mathrm{~Hz}$. Sampling was carried out in a controlled environment to make sure that reading of all sensors takes place in a similar setting. Throughout the measurement, the RAM memory did not overflow so that there was no local congestion.

The measurements were subject to three types of errors (uncertainties): calibration, random placement (some sensors were not placed near the event of interest), and random orientation (alignment). ${ }^{2}$ Fig. 1 displays an example of a combined error that arises from orientation and calibration differences between two sensor nodes measuring one and the same movement. The aim of this paper is to quantify and qualitatively describe the degree of indifference of the features considered to these types of errors.

\section{A. Measurements}

In this subsection, we describe the different types of movements we considered for our analysis. Each of these movements

\footnotetext{
${ }^{1}$ In speech recognition the spectral-roll is expressed as $\mathrm{SRO}_{\text {speech }}=$ $(\alpha / 100) \sum_{n=1}^{N} M_{t}[n]$, where $\alpha$ as a rule equals $85 \%$.

${ }^{2}$ There are, of course, other sources of errors, but we do not consider them here.
} 


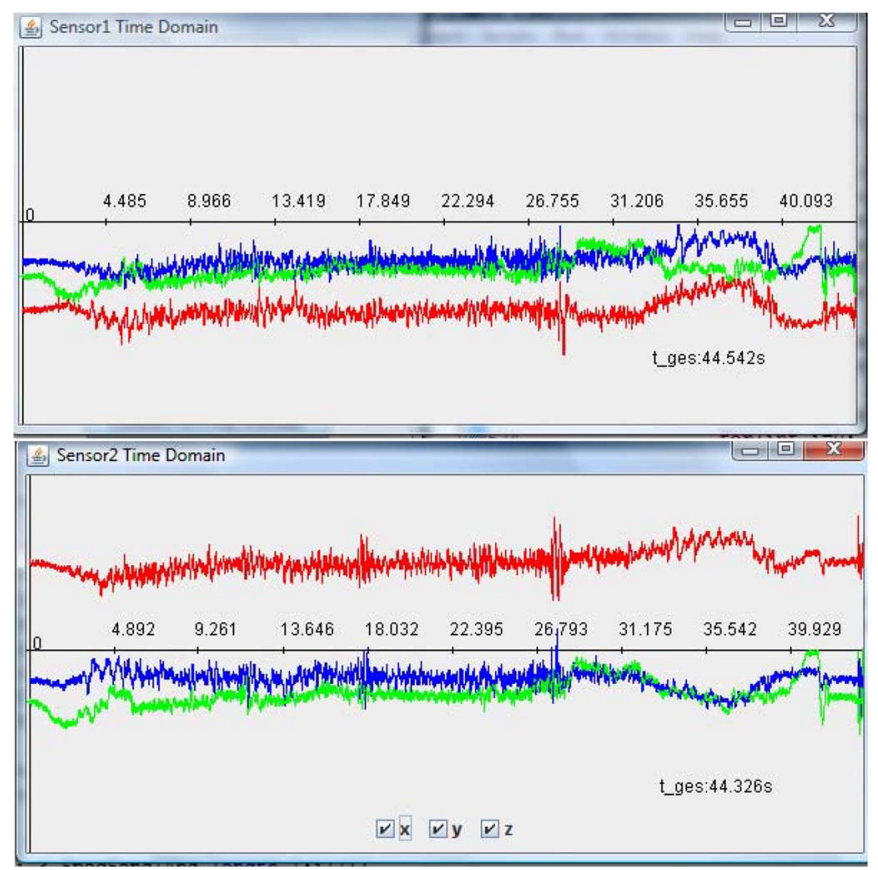

Fig. 1. Snapshot of two time-series measurements taken by two different nodes measuring the same movement. The two sensor nodes have different calibrations and placements. In both graphs, the $\mathrm{x}$-axis represents time, and the $\mathrm{y}$-axis the magnitude of acceleration.

was captured by at least two SunSpot sensor nodes in different placement settings. The nodes directly transmit the raw data to a nearby base station. Each packet was acknowledged; in case of a lost or corrupted packet, retransmission was requested and granted.

1) Movement of People:

- A single person: Two sensor nodes were placed at the opposite thighs and wrists of a walking person. The measurements were taken from calibrated sensors, but each time, they were placed randomly and they had random orientation. ${ }^{3}$

- Two persons climbed up and down a staircase, side by side (without synchronization of steps): Two sensor nodes were randomly placed in the thighs and wrists of each test person. The persons climbed a staircase of 18 steps side by side. After the climb was over, the persons turned back and climbed down the staircase. The two types of movements were labeled independently.

- Two persons climbed up and down a staircase one after another (without synchronization of steps): Two sensors were attached to the test persons as described in the previous setting, but this time, one person was walking in front of the other.

- Dancing: Two sensor nodes were attached to the thighs of the test persons. They danced for about a minute. The dance was a free and uncoordinated movement (with no premeditated pattern), but the occurrence of certain body contacts signifying some distinct movements were labeled.

- Couch: Two nodes were attached at the thighs of two test persons. The experiment measured actions and reaction
TABLE II

Overview of the Measurements DuRATION AND Data Size

\begin{tabular}{|l|l|l|}
\hline Measurement & Duration [ms] & Size [Kb] \\
\hline \hline Two persons: side by side & 45826 & 365 \\
\hline Two persons: one after another & 39222 & 329 \\
\hline Dancing & 48586 & 426 \\
\hline Couch & 45848 & 418 \\
\hline Highway drive: back \& front seats & 26776 & 235 \\
\hline Highway drive: with seat belts & 44542 & 411 \\
\hline $\begin{array}{l}\text { Highway drive: } \\
\text { (acceleration lane) }\end{array}$ & 62849 & 575 \\
\hline City drive & 64172 & 572 \\
\hline
\end{tabular}

movements whenever the test persons sat down on and got up from a couch. During the analysis of the measurements, other types of movements (drinking a coffee from a mug; writing, etc.) were deliberately filtered out.

2) Movement of Car:

- Highway drive: a series of measurements were taken from two accelerometer sensor nodes that were placed on the back and front seats of a car during a highway drive, with an average speed of $120 \mathrm{kmph}$. The sensors themselves were untethered. Measurements from these sensors were taken to investigate the existence of correlation between the different parts of a car even though these parts react differently to accelerations and brakes as well as to the irregular surface on which the car drives.

- Highway drive: Two sensors were attached to the seat belts of the driver and the front passenger and were very close to the center of gravity. However, exact alignment to the center of gravity was not made.

- Free drive: In this setting, the sensors were placed in the front cabin of the car; they were calibrated and aligned to the seats of the driver and the front passenger. This measurement was used as a reference to the measurements taken in the previous settings.

- City drive: the sensors were placed inside the glove box at the front cabin of the car, untethered.

Table II summarizes the durations and size of the measurements obtained.

\section{ANALYSIS AND RESUlTS}

\section{A. Time-Domain Features}

1) Zero-Crossing Rate: Comparison of the zero-crossing rates of the correlated and uncorrelated movements is a straightforward and inexpensive process. However, a high zero-crossing rate may indicate a high frequency measurement as well as a measurement which is highly corrupted by noise. As expected, all the measurements we took exhibit strong dissimilarities due to calibration error. Even those sensors which had similar orientation and placement and measured the same movement resulted in $\pm 40 / \mathrm{s}$ zero-crossing rate due to calibration error. Substituting the zero-crossing rate by a mean value-crossing rate resulted in a deviation ranging between 2 and $15 / \mathrm{s}$ for individual axes; and only $0-2 / \mathrm{s}$ for the absolute acceleration values of the individual measurements. ${ }^{4}$ Cross test 


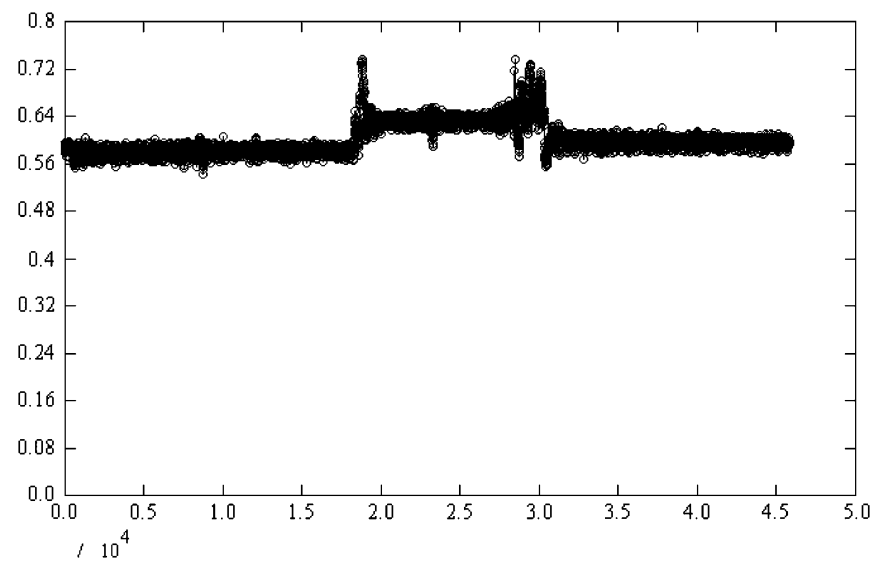

Fig. 2. Autocorrelation of the absolute acceleration values of the measurements taken from the first person.

of uncorrelated measurements, on the other hand, resulted in an overall deviation that ranges from 7 to $50 / \mathrm{s}$ for the individual axes; and 5-30/s for the absolute acceleration value.

2) Mean Value: The mean value is perhaps the simplest and the least computationally intensive feature. The deviation in value between similar (correlated) fast movements (car) for individual axes was $\pm 0.5 \mathrm{~g}$. The absolute mean value of these movements, even for randomly oriented measurements, has a markedly small deviation, i.e., $\pm 0.05 \mathrm{~g}$. On the contrary, the deviation in human movements was high. For example, the average deviation in the absolute acceleration value of similar (correlated) measurements was $\pm 0.4 \mathrm{~g}$.

3) Correlation Coefficient: The correlation coefficient in this context is a measure of the degree of similarity in movement patterns, i.e., how similar are the temporal structure of two sets of measurements. Once again, the measurements that are taken from the different parts of a car during the same drive yield a correlation between 0.4 and 0.9 . On the other hand, the correlation between dissimilar measurements was $\{0.2 \pm 0.1\}$. Exceptions to this were the measurements taken from the sensors which laid fixed on the front and back seats of the car. In which case, it was not at all possible to establish correlation. The correlation coefficients related to human movements are markedly small, i.e., in the ranges of 0.2 and 0.3 for measurements representing similar movements. The correlation coefficients of the absolute acceleration values of all the measurements are notably better. The scenario that produced the highest correlation was the movements of people on the couch. Figs. 2 and 3 show the absolute values of the correlation of the readings taken from the thighs of the two people as described in Section IV-A. As can be seen, the movement of one person produced a reaction movement in the other person.

The problem with autocorrelation is its requirement of time synchronization. In the absence of time synchronization, the correlation coefficients of all movements were very small.

4) Cross Correlation: The cross correlation is used to measure the magnitude of the time offset between two time-series measurements. This is particularly useful to model correlated movements that cannot be compared piecewise. A typical example is the correlation between the movements of people climbing up and down a staircase without synchronizing their

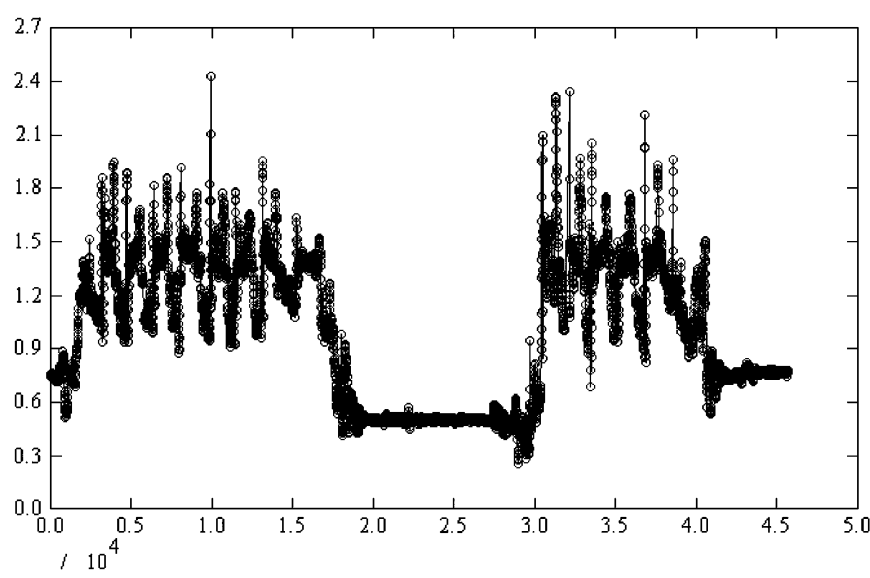

Fig. 3. Autocorrelation of the absolute acceleration values of the measurements taken from the second person.

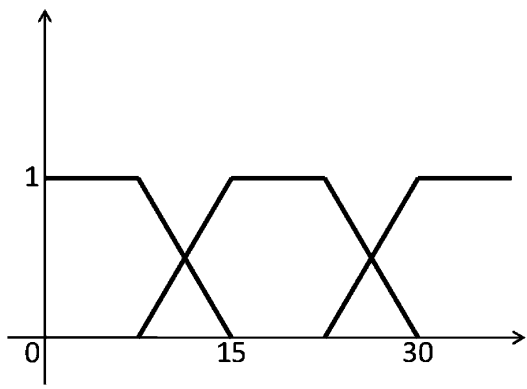

Fig. 4. Membership function for establishing the fuzzy set of the MCR feature.

steps. Intuitively, the movements should demonstrate strong correlations. However, due to the anatomy of the persons and the relative distance between the two people (back and forth), a sample-by-sample correlation was irrelevant. With the help of the cross correlation, we were able to detect and correct a mean offset value of $\pm 1.4 \mathrm{~s}$ over all the three axes of the accelerometer sensors.

5) Autocorrelation: Another approach to deal with measurements that cannot be compared piecewise is to test the linear correlation between two autocorrelation functions. This feature performs very well to test uncorrelated movements instead of correlated movements. For example, comparison of the autocorrelation of the measurements taken during the staircase movements revealed that the structure of the autocorrelation functions was almost identical even though their magnitude at any given location was different.

Table V summarizes the different time-domain features we considered. The features test both the presence and the absence of correlation between different measurements. In the second and the third column, the boundary signifies the width of the variance of similarity.

For a qualitative analysis, we defined fuzzy sets to model the uncertainty associated with each feature. We used empirical observations to define the membership function of the fuzzy sets for each feature. In each case, the membership function is defined as a trapezoid function in which the beginning and the end of the trapezoids were taken from the experiment results. The trapezoid function for the MCR is shown in Fig. 4. Equation (8) 
TABLE III

MEMBERSHIP FUNCTION DEFINITION FOR THE MCR

\begin{tabular}{|l|l|}
\hline Description & Membership Function \\
\hline \hline Low & $\mu_{l}: T(x, 0,4,1,11), m_{a}=0 ; m_{b}=15$ \\
Midium & $\mu_{m}: T(x, 17,22,14,14), m_{a}=10 ; m_{b}=30$ \\
High & $\mu_{h}: T(x, 30, \infty, 14,1), m_{a}=25 ; m_{b}=\infty$ \\
\hline
\end{tabular}

TABLE IV

MEMBERSHIP FUNCTION DEFINITION FOR THE LINEAR CORRELATION COEFFICIENTS

\begin{tabular}{|l|l|}
\hline Description & Membership Function \\
\hline \hline Low & $\mu_{l}: T(x, 0.15,0.25,0.05,0.05), m_{a}=0.1 ; m_{b}=0.3$ \\
Midium & $\mu_{m}: T(x, 0.35,0.45,0.1,1.5), m_{a}=0.25 ; m_{b}=0.6$ \\
High & $\mu_{h}: T(x, 0.65,1.1,0.19,1.0), m_{a}=0.5 ; m_{b}=1.1$ \\
\hline
\end{tabular}

TABLE V

SUMMARY OF TIME DOMAIN FEATURES

\begin{tabular}{|c|c|c|c|}
\hline Feature & $\begin{array}{l}\begin{array}{l}\text { Deviation } \\
\text { (Correlated) }\end{array} \\
\end{array}$ & $\begin{array}{l}\text { Deviation } \\
\text { (Uncorrelated) }\end{array}$ & Uncertainty \\
\hline $\bar{~} \overline{\text { ZCR/MCR (s.T) }}$ & $0.0-15.0 / \mathrm{s}$ & $7-50 / \mathrm{s}$ & Medium \\
\hline ZCR/MCR (Abs.) & $0.0-2.0 / \mathrm{s}$ & $5.0-30.0 / \mathrm{s}$ & Low \\
\hline Mean value (s.T) & $\pm 0.5 g$ & $\pm 0.5 g$ & Unsuitable \\
\hline Mean value (Abs.) & $\pm 0.05 g$ & $\pm 0.5 g$ & Medium \\
\hline Corr. coeffi. (s.T. m) & $0.2-1$ & $<0.2$ & Medium/High \\
\hline Corr. coeffi. (s.T. c) & $0.4-1.0$ & $<0.4$ & Low \\
\hline \multicolumn{4}{|c|}{$\begin{array}{l}\text { Legend } \\
\text { s.T: Individual time series measurements }(x, y, z) \\
\text { Abs.: The absolute values of the raw sensor measurements } \\
\text { m: Human movements } \\
\text { c: Car movements }\end{array}$} \\
\hline
\end{tabular}

shows the expression of the membership function for this feature. Tables III and IV display the membership definitions of the MCR and the linear correlation coefficients

$$
T\left(x, m_{1}, m_{2}, \alpha, \beta\right)=\left\{\begin{array}{ll}
0 & x<m_{a}, x \geq m_{b} \\
1 & m_{1} \leq x<m_{2} \\
\frac{\left(x-m_{a}\right)}{\alpha} & m_{a} \leq x<m_{1} \\
\frac{\left(m_{b}-x\right)}{\beta} & m_{2}<x<m_{b}
\end{array} .\right.
$$

\section{B. Frequency-Domain Features}

The frequency-domain features were extracted after transforming the time-series measurements in their entirety into frequency domain using the FFT. Exception to this is the short-time Fourier transform (STFT), in which case, the time series measurements were divided into several overlapping short frames before they were transformed into frequency-domain features.

All the frequency-domain features require preprocessing. The resource consumption of these steps is discussed in more detail in [6] and [19]. Additional to these processes, we carry out frequency normalization using a hamming window, so that the frequency resolution for each measurement is optimized.

1) Maxima: The n-maxima of a frequency spectrum was used to compare the dominant frequencies of different measurements. To obtain a significant size of representative frequency samples, first the $i$-th Maxima were summed up and divided by the total Maxima. Once this was done, comparison was made by selecting the $n$-th Maxima and observing the deviation from the average Maxima.
For human movements, $n=100$ was sufficient, while for car $n$ should be in the order of 1000 . This is because human movements contain low frequency components. The average distance between the first 100 Maxima of the individual axis for similar movements lied between 0.7 and $3 \mathrm{~Hz}$, while it was between 0.7 and $3.5 \mathrm{~Hz}$ for the absolute value of the individual axes. On the other hand, the same distance for uncorrelated (dissimilar) movements was between 1.4 and $3.9 \mathrm{~Hz}$ for the individual axes and between 1.6 and $4.8 \mathrm{~Hz}$ for the absolute value. For car movement and with the distance between the first 1000 Maxima, the corresponding results were between $5-13 \mathrm{~Hz}$ for strong correlation and $20-30 \mathrm{~Hz}$, for uncorrelated (individual dimensions); and $7-18 \mathrm{~Hz}$ and $11-25 \mathrm{~Hz}$ for absolute values.

2) Energy: The spectrum energy of a set of sensor readings reveals the spectrum's structure. In this context, the spectrum energy refers to the overall energy of the two readings being analyzed. To start with, the spectrum was divided into $n$ subbands and the portion of energy in each band was normalized by the overall energy of the spectrum. Correlation test was performed subband by subband comparison on different measurements- the stronger the correlation between the measurements, the lesser the difference between the subband energies. As a result the average difference of the subband energies was used to measure correlation. In our analysis, the energy mass of similar movements of objects was between 0.006 and 0.1 , while for uncorrelated, dissimilar movements, the mass was between 0.2 and 0.9 . Human movement was very difficult to categorize with the energy mass as the range was not strikingly different for similar and dissimilar movements.

3) Linear Correlation Coefficient: Ideally, if the spectral structures of two sets of measurements are similar, then there is a strong correlation between them. The frequency-domain linear correlation coefficients examine this hypothesis. Indeed the measurements taken from different places during the same type of movement revealed the existence of a strong correlation (between 0.6 and 0.99). Unfortunately, we also observed that correlation coefficients of unrelated movements frequently yielded values above 0.6. The best explanation for this is that all types of movements have high frequency components which undermine the significance of the band-pass frequencies, which are distinct from movement to movement. As a result, a large portion of the curves are similar and can wrongly be interpreted as being correlated.

We attempted to reduce this effect by quantizing the measurements. Even though there was some improvement, linear correlation coefficients in the frequency domain are the feeblest features. Moreover, the quantization level was very much dependent on the measurements being compared or tested-the bigger the pick to pick individual amplitudes, the larger should be the quantization level.

We investigated the change of frequencies over time as a measure of correlation. For this, we used the STFT, which is computed by dividing the sensor measurements into several overlapping frames. Each frame is then Fourier transformed, and the complex result is added to a matrix, recording the magnitude and phase of each point in time and frequency domain. As a scaling factor, we summed up the correlation coefficients and divided them by the size of the frequencies being considered. The 
test showed that a marked difference between the movements of people and the movements of cars. For human movements, the results were on the average between 0.1 and 0.3 , which confirms the results we got in the time domain for the same data set. Cross tests of uncorrelated movements resulted in weighted linear correlation coefficients that rangeg from 0.03 to 0.09 . This much could not be achieved in the time domain for the same data sets.

The result of car related movements were even better. While the correlation coefficients for similar movements were between 0.2 and 0.6 , for unrelated movements, these were between 0.02 and 0.1 . This observation suggests that other frequency-domain analysis, such as coherence, can be more expressive if they consider STFT instead of the FFT.

4) Spectral Roll-Off: The spectral roll-off is another structural feature in the frequency domain in which only the Fourier transformation of the acceleration vectors was taken into account. In most cases, such as in speech recognition, it is usually customary to consider $\alpha=85 \%$. This, however, did not produce any significant difference between correlated and uncorrelated movements. Not unexpectedly, a significant portion of the energy of most movements was contained within the lower frequency components. Subsequently, we lowered down the value of $\alpha$ to $60 \%$. As a result, similar movements revealed a roll-off distance that ranged from $0-4 \mathrm{~Hz}$ while the roll-off distance for uncorrelated movements were between 2.5 and $10 \mathrm{~Hz}$.

5) Spectral Centroid: The spectral centroid is similar to the "first n-Maxima" and indicates the relative location of the "center of gravity of the spectrum. It is computed as the weighted mean of the frequencies - the magnitudes of the frequencies being taken as weights. This scheme requires a precise knowledge of the movements being considered. The analysis was carried out by dividing the main frame into several subbands and the centroid of each subbands was independently computed and piecewise comparison was performed. The mean difference of the subband centroids, dis, is summarized by (9) as

$$
d i s=\frac{1}{N} \cdot \sum_{i=0}^{N}\left|S C_{1}(i)-S C_{2}(i)\right|
$$

where $S C_{1}$ and $S C_{2}$ refers to the spectral centroid of sensor 1 and 2, respectively; and $N$ is the number of subbands.

Regardless of the movement types, division of the entire spectrum into five equal segments resulted in a centroid distance that ranged from 0.2 to $0.5 \mathrm{~Hz}$ for related movements; and from 0.3 to $1.7 \mathrm{~Hz}$ for unrelated movements. By taking the absolute values of the spectrum, the related movements yielded a better correlation, the centroid being tighter than the previous, i.e., from 0.2 to $0.3 \mathrm{~Hz}$.

6) Spectral Flux: The spectral flux is a measure of how quickly the power spectral changes. Ideally, similar movements should have a deviation of 0 flow. To compute the spectral flux of two measurements, both should have the same time duration. In the simplest case of considering the entire duration, we obtained a spectral flux that ranged from 0.0026 to 0.25 for similar movements -0.25 is rather the worst case. Otherwise, the spectral flux of similar movements was between 0 and 0.1. On the contrary, the spectral flux of unrelated movements varied from 0.2 to 1.0 .
TABLE VI

SUMMARY OF THE FREQUENCY-DOMAIN FEATURES

\begin{tabular}{|l|l|l|l|}
\hline Feature & $\begin{array}{l}\text { Deviation } \\
\text { (Correlated) }\end{array}$ & $\begin{array}{l}\text { Deviation } \\
\text { (Uncorrelated) }\end{array}$ & Uncertainty \\
\hline \hline Maxima (s.T. m) & $0.7-3 \mathrm{~Hz}$ & $1.4-3.9 \mathrm{~Hz}$ & High \\
\hline Maxima (Abs. m) & $0.7-3.5 \mathrm{~Hz}$ & $1.6-4.8 \mathrm{~Hz}$ & High \\
\hline Maxima (s.T. c) & $5-13 \mathrm{~Hz}$ & $20-30 \mathrm{~Hz}$ & Medium \\
\hline Maxima (Abs. c.) & $7-18 \mathrm{~Hz}$ & $11-25 \mathrm{~Hz}$ & Medium \\
\hline Energy (Abs. m.) & $0.0-0.02$ & $0.02-0.9$ & Medium \\
\hline Energy (Abs. c.) & $0.0-0.1$ & $0.2-0.9$ & Low \\
\hline Corr. (FFT) s.T. Abs. & $0.6-0.9$ & $0.6-0.9$ & Very high \\
\hline Corr. (STFT) Abs. m. & $0.1-0.3$ & $0.03-0.09$ & Low \\
\hline Corr. (STFT) Abs. c. & $0.2-0.6$ & $0.02-0.1$ & Low \\
\hline Spectral roll-off (Abs.) & $0-4 \mathrm{~Hz}$ & $2.5-10.0 \mathrm{~Hz}$ & Medium \\
\hline Spectral centroid (s.T) & $0.2-0.5 \mathrm{~Hz}$ & $0.3-1.7 \mathrm{~Hz}$ & Low \\
\hline Spectral Centroid (Abs.) & $0.2-0.3 \mathrm{~Hz}$ & $0.3-1.7 \mathrm{~Hz}$ & Low \\
\hline Spectral flux (Abs.) & $0.0-0.25$ & $0.2-1.0$ & Low \\
\hline $\begin{array}{l}\text { Legend } \\
\text { s.T: Individual time-series measurements (x, y, z) } \\
\text { Abs.: The absolute values of the raw sensor measurements } \\
\text { m: Human movements } \\
\text { c: Car movements }\end{array}$
\end{tabular}

Table VI summarizes our observation for the frequency-domain analysis.

\section{CONCLUSIONS}

We investigated the expression power of several time- and frequency-domain features in the presence of calibration, placement and orientation errors. The measurements from which the features are extracted represent movements of humans (slow movements) and cars (fast movements) for various scenarios, both in calibrated and un-calibrated conditions. The time-domain features we considered were zero-crossing rate (meanvalue crossing rate), correlation coefficients, and cross correlations. The frequency-domain features were Maxima and energy; correlation coefficients of FFT and STFT, spectral roll-off, spectral centroids, and spectral flux. We observed that the features extracted from the absolute values of the raw measurements were more robust to noise and calibration errors than the features extracted directly from the raw measurements of individual axes. The frequency-domain features that were least vulnerable to noise and exhibit the strongest expression power were the correlation coefficients of the absolute values of the STFTs. The features extracted from slow movements were in general prone to measurement errors.

In the future, we aim to extend our studies by employing different types of sensors. We have already collected a large amount of data with MicaZ sensors. This will enable us to evaluate how feature extraction can be affected by node architecture.

\section{REFERENCES}

[1] S. Kim, S. Pakzad, D. Culler, J. Demmel, G. Fenves, S. Glaser, and M. Turon, "Health monitoring of civil infrastructures using wireless sensor networks," in Proc. 6th ACM Int. Conf. Information Processing in Sensor Networks, New York, 2007, pp. 254-263.

[2] P. Mohan, V. N. Padmanabhan, and R. Ramjee, "Nericell: Rich monitoring of road and traffic conditions using mobile smartphones," in Proc. 6th ACM Conf. Embedded Network Sensor Systems, New York, 2008, pp. 323-336. 
[3] M. Malinowski, M. Moskwa, M. Feldmeier, M. Laibowitz, and J. A. Paradiso, "Cargonet: A low-cost micropower sensor node exploiting quasi-passive wakeup for adaptive asychronous monitoring of exceptional events," in Proc. 5th ACM Int. Conf. Embedded Networked Sensor Systems, New York, 2007, pp. 145-159.

[4] S. A. Ballegaard, T. R. Hansen, and M. Kyng, "Healthcare in everyday life: Designing healthcare services for daily life," in Proc. 26th Аnnu. SIGCHI Conf. Human Factors in Computing Systems, New York, 2008, pp. 1807-1816.

[5] G. Werner-Allen, K. Lorincz, M. Welsh, O. Marcillo, J. Johnson, M. Ruiz, and J. Lees, "Deploying a wireless sensor network on an active volcano," IEEE Internet Comput., vol. 10, no. 2, pp. 18-25, Mar./Apr. 2006.

[6] W. Dargie, "Adaptive audio-based context recognition," IEEE Trans. Syst., Man, Cybern. A: Syst., Humans, vol. 39, no. 4, pp. 715-725, Jul. 2009.

[7] X. Chao, W. Dargie, and G. Lin, "Energy model for H2S monitoring wireless sensor network," in Proc. 2008 11th IEEE Int. Conf. Computational Science and Engineering, Washington, DC, 2008, pp. 402-409.

[8] D. Ganesan, S. Ratnasamy, H. Wang, and D. Estrin, "Coping with irregular spatio-temporal sampling in sensor networks," SIGCOMM Comput. Commun. Rev., vol. 34, no. 1, pp. 125-130, 2004.

[9] S. Lin, "Data Management and Data Analysis Techniques for Wireless Sensor Networks," Ph.D. dissertation, Univ. California, Riverside, CA, 2007.

[10] C. Tang and C. S. Raghavendra, Compression Techniques for Wireless Sensor Networks pp. 207-231, 2004.

[11] S. Bandyopadhyay, Q. Tian, and E. J. Coyle, "Spatio-temporal sampling rates and energy efficiency in wireless sensor networks," IEEE/ACM Trans. Netw., vol. 13, no. 6, pp. 1339-1352, Dec. 2005.

[12] E. F. Nakamura, A. A. F. Loureiro, and A. C. Frery, "Information fusion for wireless sensor networks: Methods, models, and classifications," ACM Comput. Surv., vol. 39, no. 3, p. 9, 2007.

[13] X. Dai, F. Xia, Z. Wang, and Y. Sun, "A survey of intelligent information processing in wireless sensor network," Mobile Ad-Hoc Sensor Netw., pp. 123-132, 2005.

[14] T. Huynh and B. Schiele, "Analyzing features for activity recognition," in Proc. ACM 2005 Joint Conf. Smart Objects and Ambient Intelligence, New York, 2005, pp. 159-163.

[15] J. A. Ward, P. Lukowicz, G. Troster, and T. E. Starner, "Activity recognition of assembly tasks using body-worn microphones and accelerometers," IEEE Trans. Pattern Anal. Mach. Intell., vol. 28, no. 10, pp. 1553-1567, Oct. 2006.

[16] M. Engin, S. Demiră̆, E. Z. Engin, G. Çelebi, F. Ersan, E. Asena, and Z. Çolakoğlu, "The classification of human tremor signals using artificial neural network," Expert Syst. Appl., vol. 33, no. 3, pp. 754-761, 2007.

[17] N. L. W. Keijsers, M. W. I. M. Horstink, and S. C. A. M. Gielen, "Ambulatory motor assessment in parkinson's disease," Movement Disorder Soc., vol. 21, no. 1, pp. 34-44, 2006.

[18] R. Marin-Perianu, J. Hurink, and P. Hartel, "A generalized clustering algorithm for dynamic wireless sensor networks," in Proc. 2008 IEEE Int. Symp. Parallel and Distributed Processing with Applications, Washington, DC, 2008, pp. 863-870.
[19] W. Dargie and T. Tersch, "Recognition of complex settings by aggregating atomic scenes," IEEE Intell. Syst., vol. 23, no. 5, pp. 58-65, Sep. 2008.

[20] A. J. Eronen, V. T. Peltonen, J. T. Tuomi, A. P. Klapuri, S. Fagerlund, T. Sorsa, G. Lorho, and J. Huopaniemi, "Audio-based context recognition," IEEE Trans. Audio, Speech, Lang. Process., vol. 14, no. 1, pp. 321-329, Jan. 2006.

[21] W. Dargie, "Analysis of time and frequency domain features of accelerometer measurements," in Proc. 3rd IEEE Workshop on Performance Modeling and Evaluation of Computer and Telecommunication Networks (PMECT 2009), 2009.

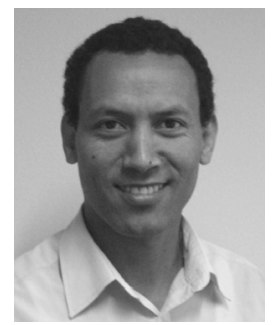

Waltenegus Dargie (M'08) received the B.Sc. degree from the Nazareth Technical College, Nazareth, Ethiopia, in 1997, and the M.Sc. degree from the Technical University of Kaiserslautern, Kaiserslautern, Germany, in 2002, both in electrical engineering, and the Ph.D. degree in computer engineering from the Technical University of Dresden, Dresden, Germany, in 2006.

$\mathrm{He}$ is a Researcher/Lecturer at the Technical University of Dresden. Previously, he was a Researcher with both the Department of Electrical Engineering and Computer Science, University of Kassel, Kassel, Germany, between 2002 and 2005 and the Fraunhofer Institute of Experimental Software Engineering, Kaiserslautern, Germany, between 2002 and 2003. His research interests include autonomous computing, context-aware computing, wireless networks, and digital signal processing.

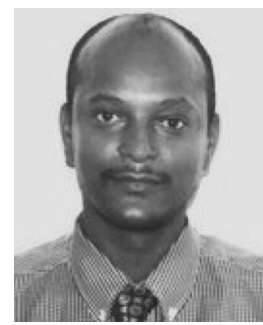

Mieso K. Denko (SM'07) received the M.Sc. degree from the University of Wales, U.K., and the Ph.D. degree from the University of Natal, Natal, South Africa, both in computer science.

Currently, he is with the School of Computer Science, University of Guelph, Guelph, ON, Canada. His current research interests include wireless networks, mobile and pervasive computing, wireless mesh networks, body area sensor networks and network security. He has co-edited three books in the areas of pervasive computing, autonomic computing and wire-

less networking.

Dr. Denko is a founder/co-founder of a number of ongoing international workshops \& symposia and served as Program Chair/Co-Chair for several IEEE/ACM/IFIP international conferences. Currently, he is Guest Co-Editor of Special Issues for several journals, including the ACM/Springer Mobile Networks and Applications (MONET) and the IEEE SYSTEMS JOURNAL. He is an Editorial Board Member of international journals including, the International Journal of Smart Homes (IJSH), the Journal of Ubiquitous Computing and Communications (UBICC), and is an Associate Editor of the International Journal of Communication Systems (IJCS), Wiley, Security \& Communications Network (SCN), Wiley, and the Journal of Ambient Intelligence and Humanized Computing (JAIHC), Springer. He is a Senior Member of the ACM. 